\title{
Improving super-resolution mapping through combining multiple super-resolution land-cover maps
}

\author{
Xiaodong $\mathrm{Li}^{\mathrm{a}}$, Feng Ling ${ }^{\mathrm{a} *}$,Giles M. Foody ${ }^{\mathrm{b}}$, Yun Du ${ }^{\mathrm{a}}$
}

a. Key laboratory of Monitoring and Estimate for Environment and Disaster of Hubei province, Institute of Geodesy and Geophysics, Chinese Academy of Sciences, Wuhan 430077, PR China; b. School of Geography, University of Nottingham,

University Park, Nottingham NG7 2RD, U. K.

(*Corresponding author; e-mail: lingf@whigg.ac.cn)

\begin{abstract}
Super-resolution mapping (SRM) is an ill-posed problem, and different SRM algorithms may generate non-identical fine spatial resolution land-cover maps (sub-pixel maps) from the same input coarse spatial resolution image. The output sub-pixels maps may each have differing strengths and weaknesses. A multiple SRM (M-SRM) method that combines the sub-pixel maps obtained from a set of SRM analyses, obtained from a single or multiple set of algorithms, is proposed in this study. Plurality voting, which selects the class with the most votes, is used to label each sub-pixel. In this study, three popular SRM algorithms, namely, the pixel swapping algorithm (PSA), the Hopfield neural network (HNN) algorithm, and Markov random field (MRF) based algorithm, were used. The proposed M-SRM algorithm was validated using two data sets: a simulated multi-spectral image and an airborne visible/infrared imaging spectrometer (AVIRIS) hyperspectral image. Results show that the highest overall accuracies were obtained by M-SRM in all experiments. For example, in the AVIRIS image experiment, the highest overall accuracies of PSA, HNN and MRF were $88.89 \%, 93.81 \%$ and $82.70 \%$ respectively, and increased to $95.06 \%, 95.37 \%$ and $85.56 \%$ respectively for M-SRM obtained from the multiple PSA, HNN and MRF analyses.
\end{abstract}

Keywords: super-resolution land-cover mapping; mixed pixels; voting 


\section{Introduction}

Super-resolution mapping (SRM) is a process used to predict the spatial distribution of land-cover classes in image pixels at a finer spatial resolution than that of the input data. As such, SRM has an important role to play in reducing the mixed pixel problem that is commonly encountered in mapping land-cover from remotely sensed data. A variety of SRM methods are available and often employ constraints to guide the analysis to an appropriate solution (Foody and Doan 2007; Foody, Muslim, and Atkinson 2005; Ge 2013; Ge et al. 2014; Hu et al. 2015; Li, Ling, and Du 2012; Ling et al. 2013; Ling et al. 2010; Wang, Wang, and Liu 2012). For example, an analysis may be constrained to ensure that the land-cover class areal proportions for a coarse resolution pixel, estimated by a soft classification, are maintained within the geographical area it represents and/or that prior information on the spatial pattern of the land-cover is used to generate the sub-pixel land-cover map. However, the solution space of SRM is large, and it provides multiple plausible solutions that satisfy the constraints. Previous studies have shown that a varied set of land-cover representations may arise from the same coarse spatial resolution image through the use of different SRM methods (Foody and Doan 2007; Makido, Messina, and Shortridge 2008). Typically, the identification of an optimal SRM method in advance is a difficult, if not impossible, challenge.

The multiple classifier system is a powerful solution to difficult pattern recognition problems involving large class sets (Ho, Hull, and Srihari 1994), and this system has shown considerable potential to increase the accuracy of classifications of remotely sensed imagery (Benediktsson and Sveinsson 2003; Briem, Benediktsson, and Sveinsson 2002; Bruzzone, Cossu, and Vernazza 2004; Kavzoglu and Colkesen 2013). Since each classifier usually generates a unique land-cover map that satisfies the classifier's objective function, a set of different maps may be generated from a suite of classifiers. The multiple classifier system combines the set of maps, aiming to produce a final map that is of superior quality to the individual maps it is made from. Although the multiple classifier system has been extensively investigated for the classification of remotely sensed imagery, it has been mostly used to combine multiple land-cover maps generated with conventional (hard) image classifications at the pixel scale. As the latter type of analysis may be degraded by the mixed pixel problem, the multiple classifier approach may, however, also be used to combine 
multiple soft classifications (Doan and Foody 2007). Although soft classification can predict sub-pixel scale class areal proportion information it does not indicate the geographical location of the classes within the area of each coarse resolution pixel. A simple enhancement would be to generate a set of sub-pixel maps from the soft classifications via a series of SRMs and combine them. Little research has, however, focused on the ensemble of multiple SRM algorithms. Many studies show that no single SRM algorithm can be expected to perform perfectly, and each SRM output has its own strengths and weaknesses (Atkinson 2009; Ling et al. 2014). The combination of multiple SRM outputs could utilize the different information of each while addressing drawbacks of the individual methods, and this combination is expected to produce a more accurate sub-pixel map than that produced by an individual SRM algorithm.

The use of different SRM algorithms, or a single algorithm with, for example, dissimilar parameter settings, allows the generation of non-identical sub-pixel maps from the same data (Makido, Messina, and Shortridge 2008). In this study, the multiple SRM (M-SRM) approaches that combine the multiple maps from a single SRM algorithm and from multiple SRM algorithms are explored. Three popular SRM algorithms, namely, the pixel swapping algorithm (PSA) (Atkinson 2005), the Hopfield neural network (HNN) algorithm (Su et al. 2012a; Tatem et al. 2001), and the Markov random field (MRF)-based algorithm (Kasetkasem, Arora, and Varshney 2005; Li, Du, and Ling 2012), were used. The combination of multiple sub-pixel maps obtained from a set of SRM analyses was accomplished with a voting based approach. The proposed M-SRM was validated using two data sets: a simulated multi-spectral image and an airborne visible/infrared imaging spectrometer (AVIRIS) hyperspectral image. Moreover, analyses using different parameter settings for each algorithm were undertaken allowing the combination process to be based upon outputs from a single algorithm or multiple algorithms.

\section{Method}

\subsection{Component SRM algorithms}

\subsubsection{SRM algorithms introduction}


Three popular SRM algorithms, the PSA, HNN and MRF, were adopted. In these methods, the coarse resolution pixel is broken down to sub-pixels (fine resolution pixels) initially, and the different algorithms have dissimilar strategies to label the sub-pixels. This section outlines the salient features of each.

The PSA is applied to a soft classification output. It is designed to convert the class areal proportions predicted by a soft classification into a set of (hard) sub-pixel land-cover class allocations. This is achieved by swapping sub-pixel class labels in a way that maximizes the spatial autocorrelation between neighbouring sub-pixels under the constraint that the original class areal proportions for the area represented by each coarse resolution pixel are maintained (Atkinson 2005). If swapping a pair of sub-pixels in a coarse resolution pixel would increase the spatial autocorrelation of the output map, the sub-pixels are swapped. Otherwise, no swap is made. The PSA is converged until no swap of sub-pixels is made or a pre-defined iteration is reached. This approach is reasonable when the land-cover exists as a mosaic of patches that are larger than the size of the coarse resolution pixel (Atkinson 2009). The class areal proportions are unchanged before and after each swapping of sub-pixels in the coarse pixel.

The HNN is also applied to a soft classification output. The HNN is a recurrent neural network and is formulated as an energy minimization tool to predict the sub-pixel land-cover distribution within the geographical area of each coarse resolution pixel (Tatem et al. 2001). By utilizing information contained in surrounding pixels, the land-cover within each pixel may be mapped using a simple spatial clustering function coded into the HNN. In the HNN-based SRM, sub-pixels are allocated (hard) land-cover class labels in a manner that reflects directly the class areal proportions predicted by a soft classification. The relative weights of a set of goal functions control the nature of the final output. The HNN class areal proportions constraint aims to retain the class areal proportional information output from the soft classification that informs the SRM. The class areal proportions outputted from soft classification do not have to be faithfully maintained in the sub-pixel map, depending on the weight of the class areal proportions constraint in the HNN goal function.

The MRF-based SRM is applied directly on the remotely sensed imagery, and is thus different from the PSA and HNN. The MRF-based SRM goal function is not 
relevant to the class areal proportions directly, but is modeled by analyzing the image spectral information and the land-cover spatial information (Kasetkasem, Arora, and Varshney 2005). The MRF-based SRM goal function includes an image spectral constraint and a land-cover spatial constraint. The spectral constraint is the assumption that the coarse pixel has a spectral response that is generated from the combined spectra from the classes contained in the sub-pixel map. The spectral constraint aims to refine the sub-pixel labels in order that the degraded and observed coarse resolution pixel spectra are similar (Tolpekin and Stein 2009). In the spatial constraint, it is assumed that a sub-pixel map has MRF properties, and the land-cover class occupying neighbouring sub-pixels are more likely to come from the same class than different classes. The MRF-based SRM land-cover spatial constraint is similar to that adopted in the PSA and HNN which maximizes the spatial autocorrelation between neighbouring sub-pixels in the result sub-pixel map.

\subsubsection{SRM map initialization}

The PSA and HNN use the class areal proportions generated from the soft classification as input and aim to maintain the class areal proportions in the result sub-pixel map, whereas the MRF is applied directly to the original remotely sensed image. The final sub-pixels land-cover map is generated by the SRM analysis using an iteratively refined fine resolution map that is provided, along with the class areal proportions or the original remotely sensed image, to the PSA, HNN and MRF algorithms. The initial value at each sub-pixel location will have an effect on the SRM performance, and different initialization maps may result in dissimilar SRM outputs (Makido, Messina, and Shortridge 2008).

The PSA initialization map is generated based on the soft classification output. The PSA initialization map is a sub-pixel land-cover map, and each sub-pixel is given an initial class value of $c(c \in 1, \cdots, C$, and $C$ is the number of land-cover classes). The PSA initialization map is produced by randomly assigning sub-pixels class labels in a manner that maintains the class proportion information conveyed by the prior soft classification (Atkinson 2005). The MRF initialization map is also a land-cover map, and can be generated based on the soft classification output or without using the soft classification output by assigning each sub-pixel label randomly within the range 1 to $C$. The initial sub-pixel map based on soft classification output is an appropriate 
starting point to result in a faster convergence of the MRF algorithm (Kasetkasem, Arora, and Varshney 2005). The HNN initialization map is not hard-classified land-cover maps but soft-classified class areal proportions, and is generated without using the soft classification output (Tatem et al. 2001). The $C$ class proportion images are represented by $C$ interconnected layers, and the neurons within these layers are referred to by coordinate notation at the sub-pixel scale. An iterative analysis is then undertaken in which the neurons ultimately indicate the class label for each sub-pixel given the goal constraints applied.

\subsection{M-SRM}

In the M-SRM, the combination of multiple sub-pixel maps obtained from a set of SRM analyses was accomplished via voting. Voting is a simple rule for combining the outputs of multiple estimators by treating the output of each estimator as a vote. There are many voting strategies that may be implemented such as plurality voting, weighted voting and soft voting (Latif-Shabgahi, Bass, and Bennett 2004; Parhami 1994). Plurality voting (Lin et al. 2003) is a combination strategy that selects the candidate with the most votes, assuming that the choice with the most votes should be the best choice. Plurality voting is conducted on the basis that the decision of a group result is superior to that of a single individual, and is one of the most extensively used combination strategies and can achieve a preferable trade-off between identification and rejection rates. Plurality voting was used here to select the class label for each sub-pixel from the multiple SRM outputs available.

The voting procedure can be illustrated for an analysis of a coarse spatial resolution remotely sensed image that contains $I \times J$ pixels. The SRM generated from the latter image is a fine resolution land-cover map (sub-pixel map) with $I \times s \times J \times s$ pixels, where $s$ is the scale factor and each coarse resolution pixel contains $s^{2}$ sub-pixels. Each sub-pixel is labelled with one of the $C$ classes, with $c\left(\alpha_{h, i, j}\right)$ be the class label of $\alpha_{h, i, j}\left(c\left(\alpha_{h, i, j}\right) \in 1, \cdots, C\right)$ where $\alpha_{h, i, j}$ is the $h^{\text {th }}\left(h=1, \cdots, s^{2}\right)$ sub-pixel in the coarse resolution pixel $(i, j)(i=1, \cdots, I, j=1, \cdots, J)$. Assume $K$ SRM algorithms are used in M-SRM. Let $V_{k}\left(c\left(\alpha_{h, i, j}\right)=c\right)$ be the vote of class $c$ for sub-pixel $\alpha_{h, i, j}$ from the $k^{\text {th }}(k=1, \cdots, K)$ SRM algorithm. The predicted class for 
sub-pixel $\alpha_{h, i, j}$ in M-SRM obtained with plurality voting is derived by maximizing the following function:

$$
\underset{c}{\arg \max }\left(\sum_{k=1, \cdots, K} V_{k}\left(c\left(\alpha_{h, i, j}\right)=c\right)\right)
$$

The use of different initialization maps may result in dissimilar SRM outputs. In order to explore the influence of different initialization maps on M-SRM, each SRM algorithm for M-SRM combination is run a number of predefined times with different initialization maps. Each SRM is run $N$ times, and the vote $V_{k}\left(c\left(\alpha_{h, i, j}\right)=c\right)$ is related with the $N$ sub-pixel maps from the $k^{\text {th }}$ SRM algorithm. The label of a sub-pixel $\alpha_{h, i, j}$ in M-SRM can be dependent on the classes depicted in the multiple maps for that sub-pixel, thus the vote $V_{k}\left(c\left(\alpha_{h, i, j}\right)=c\right)$ is determined according to the number of times that the sub-pixel $\alpha_{h, i, j}$ is labelled as class $c$ from the $N$ times of the $k^{\text {th }} \operatorname{SRM}(k=1, \ldots, K)$ as:

$$
V_{k}\left(c\left(\alpha_{h, i, j}\right)=c\right)=\sum_{n=1, \cdots, N} \delta\left(c_{k, n}\left(\alpha_{h, i, j}\right), c\right)
$$

where $c_{k, n}\left(\alpha_{h, i, j}\right)$ is the label of $\alpha_{h, i, j}$ from the $n^{\text {th }}(n=1, \cdots, N)$ result derived from the $k^{\text {th }} \mathrm{SRM}$ algorithm; $\delta\left(c_{k, n}\left(\alpha_{h, i, j}\right), c\right)$ is the Kronecker delta function that equals to 1 if $c_{k, n}\left(\alpha_{h, i, j}\right)=c$ and 0 otherwise. This ensemble approach of M-SRM, called the pixel-based M-SRM, processes the labels of each sub-pixel from the multiple SRM outputs without considering the autocorrelation between spatially adjacent sub-pixels.

Spatial context captures spatial information relative to local features in an image, and has been used in the improving of image classification accuracy (Tarabalka et al. 2010). Spatial context can be described in terms of relations of neighbouring objects. It creates connections among pixels, and can be used to investigate the spatial autocorrelation between spatially close pixels. The basis is that sub-pixels that are close together are more likely to be similar in labeling than those that are far apart. With the context information, the problem of speckling (i.e. individual pixels differing in class label from their surrounding pixels) is reduced in image classification. In the 
aforementioned pixel-based M-SRM, the ensemble of different SRM outputs depends only on the labels of a sub-pixel in the multiple outputs but ignores the spatial context information for that sub-pixel, and the speckling problem may affect the M-SRM accuracy. A context-based M-SRM that incorporates the spatial context information among neighbouring sub-pixels in the available SRM outputs is proposed and expected to minimize the speckling problem. The context-based M-SRM, in which the labeling of each sub-pixel is related to the labels of neighbouring sub-pixels, is designed as follows.

Define $\eta\left(\alpha_{h, i, j}\right)$ as the sub-pixel neighbourhood that includes all sub-pixels inside a square window of $W \times W$ sub-pixels centred on $\alpha_{h, i, j}$. The neighbourhood window size $W$ is the length of the square side, and can be set to $1,3,5,7$, or any other odd integer. Assume $\alpha_{l}$ is a neighbourhood sub-pixel of $\alpha_{h, i, j}$ in $\eta\left(\alpha_{h, i, j}\right)$. The context-based M-SRM integrates local spatial autocorrelation between neighbourhood sub-pixels, with the magnitude of the autocorrelation inversely related to the distance between the sub-pixels under consideration. The effect of $\alpha_{l}$ on the labeling of $\alpha_{h, i, j}$ in the $W \times W$ window may be dissimilar, depending on the distance between $\alpha_{l}$ and $\alpha_{h, i, j}$. The effect of sub-pixel $\alpha_{l}$ on $\alpha_{h, i, j}$ in the $W \times W$ neighbourhood window is defined as the weight $w\left(\alpha_{h, i, j} \mid \alpha_{l}\right)$. Many distance-dependent weighting functions, including the Gaussian model, the inverse distance weighting function and the exponential decay function, can be employed to measure the variation of $w\left(\alpha_{h, i, j} \mid \alpha_{l}\right)$ with the distance between $\alpha_{l}$ and $\alpha_{h, i, j}$. The Gaussian model is adopted in this paper:

$$
w\left(\alpha_{h, i, j} \mid \alpha_{l}\right)=\exp \left(-\frac{d\left(\alpha_{h, i, j}, \alpha_{l}\right)^{2}}{r^{2}}\right)
$$

where $d\left(\alpha_{h, i, j}, \alpha_{l}\right)$ is the Euclidean distance between $\alpha_{l}$ and $\alpha_{h, i, j} ; r$ is the range value that controls the relative magnitude of $w\left(\alpha_{h, i, j} \mid \alpha_{l}\right)$ with the distance $d\left(\alpha_{h, i, j}, \alpha_{l}\right)$. The variation in the magnitude of the weight $w\left(\alpha_{h, i, j} \mid \alpha_{l}\right)$ with the 
variation of $d\left(\alpha_{h, i, j}, \alpha_{l}\right)$ according to different range value $r$ is shown in figure 1 . The weight $w\left(\alpha_{h, i, j} \mid \alpha_{l}\right)$ decreases very slowly with distance $d\left(\alpha_{h, i, j}, \alpha_{l}\right)$ when $r=10$, and the spatial autocorrelations between distant sub-pixels in the $W \times W$ window are high $\left(w\left(\alpha_{h, i, j} \mid \alpha_{l}\right)\right.$ approximates to 0.8 when $\left.d\left(\alpha_{h, i, j}, \alpha_{l}\right)=5\right)$. By contrast, the weight $w\left(\alpha_{h, i, j} \mid \alpha_{l}\right)$ decreases most sharply with distance $d\left(\alpha_{h, i, j}, \alpha_{l}\right)$ when $r=1$, and the spatial autocorrelations between distant sub-pixels in the $W \times W$ window are low $\left(w\left(\alpha_{h, i, j} \mid \alpha_{l}\right)\right.$ approximates to 0 when $\left.d\left(\alpha_{h, i, j}, \alpha_{l}\right)=5\right)$. Note that when $W=1$, the window is of size $1 \times 1$ and hence the label of a sub-pixel pixel is only dependent on the classes depicted in the multiple maps for that sub-pixel, and there is no use of contextual information in this situation. The context-based M-SRM is degraded to pixel-based M-SRM in this case.

\#Insert Figure 1 here\#

Given the $N$ sub-pixel maps from the $k^{\text {th }} \mathrm{SRM}$ algorithm and according to the neighbourhood system $\eta\left(\alpha_{h, i, j}\right)$ and the weight $w\left(\alpha_{h, i, j} \mid \alpha_{l}\right)$, the vote $V_{k}\left(c\left(\alpha_{h, i, j}\right)=c\right)$ in the context-based M-SRM is calculated as

$$
V_{k}\left(c\left(\alpha_{h, i, j}\right)=c\right)=\sum_{n=1, \cdots, N} \sum_{l=\eta\left(\alpha_{h, i, j}\right)} w\left(\alpha_{h, i, j} \mid \alpha_{l}\right) \times \delta\left(c_{k, n}\left(\alpha_{l}\right), c\right)
$$

where $c_{k, n}\left(\alpha_{l}\right)$ is the label of $\alpha_{l}$ from the $n^{\text {th }}(n=1, \cdots, N)$ result derived from the $k^{\text {th }}$ SRM algorithm.

\subsection{Accuracy assessment.}

The accuracy of each land-cover map obtained from the SRM analyses was assessed relative to a reference land-cover map of the same geographical area which has the same resolution as the SRM output and was expressed as the percentage of cases correctly allocated (i.e. overall accuracy); details of the reference maps are provided 
below for each experiment. The accuracy of the class areal proportion images unmixed from soft classification was also assessed. The reference class areal proportion images were first calculated based on the reference land-cover map, and the class areal proportion for each class in each coarse resolution pixel was calculated by dividing the number of sub-pixels of that class in the coarse pixel by the square of scale factor $\left(s^{2}\right)$. Then the unmixed and reference class areal proportion images were compared using the root mean square error (RMSE) value (Jin, Wang, and Zhang 2010):

$$
\mathrm{RMSE}=\frac{1}{C} \sum_{c=1}^{C} \sqrt{\frac{1}{I \times J} \sum_{i=1}^{I} \sum_{j=1}^{J}\left(\theta_{c, i, j}-\omega_{c, i, j}\right)^{2}}
$$

where $\theta_{c, i, j}$ and $\omega_{c, i, j}$ are the class areal proportion of class $c$ in the coarse resolution pixel $(i, j)$ in the reference land-cover map and unmixed class areal proportion image.

\section{Experiments and results}

Experiments using a simulated multi-spectral image and an AVIRIS hyperspectral image were conducted to assess the proposed M-SRM method. The PSA and MRF performances are related with the number of neighborhood sub-pixels $(L)$. When the number is 1 this means that the analysis is based upon the eight immediate sub-pixel neighbors that lie within a $3 \times 3$ window centred on the sub-pixel of interest. In the PSA and MRF, the scale factor $s$ and the number of neighbouring sub-pixels $L$ are two correlated parameters and different combination of $s$ and $L$ will yield different SRM results (Atkinson 2005; Su et al. 2012b; Tolpekin and Stein 2009). The optimal number of neighbouring sub-pixels $L$ should not be too large and no more than the scale factor $s$ (Atkinson 2005), and were set to $L=s-1$ in the PSA (Su et al. 2012b) and in the MRF (Tolpekin and Stein 2009).

The pixel-based M-SRM and context-based M-SRM were assessed. The set of M-SRM analyses undertaken are summarized in Table 1. The SRM repetition number $N$ was set to 10 , and each single SRM algorithm was performed 10 times using different initialization maps. The initialization maps are sub-pixel land-cover maps for PSA and MRF and sub-pixel soft-classified class areal proportion images for HNN. In order to fairly compare the accuracy of single PSA and MRF algorithms, the same set 
of sub-pixel initialization maps, which contained 10 different sub-pixel initialization maps, was inputted to PSA and HNN. For the context-based M-SRM, the sub-pixel neighbourhood window size $W$ was set to 3,5, 7, and 9, and the range value $r$ was set to $1,2,3$, and 10 , respectively.

\#Insert Table 1 here\#

\subsection{Simulated multi-spectral image experiment}

\subsubsection{Overview}

A simulated multi-spectral image was used to control for possible sources of endmember extraction error. A real fine resolution image was used as a starting point. Visual classification of this image yielded a ground reference map for the test site. A 5 waveband multispectral image of the site was then generated using a set of spectral endmembers generated to fit with the classes depicted in the reference map. The derived multispectral imagery was then degraded with a $5 \times 5$ pixel mean filter. A soft classification of the latter coarse spatial resolution imagery was obtained using a linear mixture model (Hu and Weng 2011; Settle and Drake 1993). The class areal proportion images generated from soft classification were used as the class proportions constraints in the PSA and HNN, and to generate the initial sub-pixel land-cover maps for PSA and MRF. The coarse resolution image was also used in the MRF spectral constraint.

\subsubsection{Data}

The starting point image was a subset of a QuickBird panchromatic image of Wuhan, Hubei Province, China (figure 2, spatial resolution of $0.6 \mathrm{~m}, 30^{\circ} 35^{\prime} 51^{\prime \prime} \mathrm{N}$ and $\left.114^{\circ} 19^{\prime} 56^{\prime \prime} \mathrm{E}\right)$. The panchromatic image was manually interpreted to yield a reference map for an area of $120 \times 120$ pixels of 4 classes identified: tree, grass, bare earth, and path. A simulated 5 band multispectral image was generated using four sets of spectral endmembers, and the digital number values of the four endmembers are [630, $425,270,130,185]^{\mathrm{T}},[210,380,130,260,310]^{\mathrm{T}},[150,590,340,560,440]^{\mathrm{T}}$, and $[400$, $220,520,360,650]^{\mathrm{T}}$. The covariance matrices were defined following the approach 
discussed in Tolpekin and Stein (2009), where the covariance matrices for all the classes were manually set to $\mathbf{M} \times A$. $\mathbf{M}$ is an identity matrix, size $B \times B$ ( $B$ is the number of spectral bands and $B=5$ in this experiment), and $A=1,200$ is a constant. The spectral response of each class was normally distributed in each waveband (Foody and Doan 2007; Tolpekin and Stein 2009).

\#Insert Figure 2 here\#

\subsubsection{Results and discussion}

The sub-pixel maps obtained from the PSA, HNN and MRF with the highest overall accuracies as well as those produced by the pixel-based M-SRM and the context-based M-SRM with highest accuracy for each analysis are shown in figure 2. In the map obtained from the PSA, many speckle-like artefacts (examples are highlighted in the red circles in figure 2) were observed. These arose from spectral unmixing errors. The linear unmixing analysis may, for example, allocate a small fractional cover of a class that is absent to a pixel and because of the constraints used in the PSA this fractional cover must be maintained in the SRM. But the representation obtained was close to the references with an RMSE for the unmixed class areal proportions of 0.0324 . Scatter plots of the reference and unmixed class areal proportions are shown in figure 3. The scatter plots indicate that many estimated class areal proportion values are close but not identical to the reference values for different classes. Unlike with the PSA that maintains the class proportional information, the HNN and MRF eliminated the speckle-like artefacts due to the spatial smoothing effect based on the spatial autocorrelation model. It was also evident that parts of the path were poorly represented, with some sections disconnected in the PSA, HNN and MRF results (highlighted in the green circle in figure 2). This is because class spatial autocorrelation, which is reasonable where the land-cover target of interest is larger than the pixel size, is adopted as the land-cover prior information in the PSA, HNN and MRF. Many parts of the linear connected path were not larger than the coarse resolution pixel size, and were smoothed and disconnected in the results. 
The outputs from the M-SRM approach differed from those obtained from the single SRM analyses. The map generated from the pixel-based M-PSA contained more connected path and less speckle-like artefacts than the map from the PSA. The maps from the pixel-based M-HNN and M-MRF showed the path to be more connected than the maps from the standard single HNN and MRF analyses. This is because errors may exist in individual output but are more frequently labelled correctly in the other maps available to the M-SRM. The context-based M-SRM integrates the neighbourhood sub-pixel information and this eliminated most speckle-like artefacts. The maps from the context-based M-HNN and M-MRF showed the path to be more fully connected than the maps from the pixel-based M-HNN and M-MRF. The maps from the pixel-based and context-based M-PSA-HNN, M-PSA-MRF, M-HNN-MRF and M-PSA-HNN-MRF showed few speckle-like artefacts and the path to be highly connected. This is because the maps obtained from different SRM algorithms were different (figure 2), and the sub-pixels labelled as speckle-like artefacts or the disconnected features from one SRM algorithm were labelled correctly in the output of other SRM algorithms.

\#Insert Tables 2-4 here\#

Tables 2, 3 and 4 show the overall accuracies of single SRM and M-SRM algorithms. With a single SRM algorithm it was evident that the combination of a set of SRMs obtained from it could yield an increase in accuracy. The highest overall accuracies of PSA, HNN and MRF were 90.14\%, 89.15\% and 89.76\% respectively, and increased to $91.52 \%, 91.38 \%$ and $91.04 \%$ respectively for the pixel-based M-PSA, M-HNN and M-MRF, and increased to $92.27 \%, 91.60 \%$ and $91.27 \%$ respectively for the context-based M-PSA, M-HNN and M-MRF.

It was also evident that the accuracy of M-SRM was influenced by several factors. First, the highest overall accuracies of the context-based M-SRM were higher than that of the pixel-based M-SRM for each M-SRM. The accuracy of the context-based M-SRM was affected by the neighbourhood window size $W$ and range value $r$. In the context-based M-SRM, a larger $W$ indicates a larger neighbourhood 
window size that explores more sub-pixels with local spatial autocorrelation, and the spatial autocorrelations between distant sub-pixels in the $W \times W$ neighbourhood window are higher with a larger $r$. In general, the context-based M-SRM with $W<5$ and $r<3$ generated the highest overall accuracy in this experiment. The reference map contains linear path objects that are not larger than the coarse spatial resolution pixel, and could be over-smoothed if the neighbourhood window $W$ is large and the spatial autocorrelations between distant sub-pixels are high with large $r$. Second, for M-SRM that combined different SRM algorithms, the algorithms selected for inclusion played a key role in determining the accuracy of the final map. The mean overall accuracy of PSA was higher than that of HNN and MRF; the overall accuracies of M-SRM that combined PSA were higher than those that excluded the PSA. Specifically, the highest overall accuracy of M-PSA was higher than that of M-HNN and M-MRF, and the highest overall accuracies of M-PSA-HNN, M-PSA-MRF and M-PSA-HNN-MRF were higher than that of M-HNN-MRF which excluded the PSA. These results highlight the importance of selecting algorithms for use in a multiple classifier system with care. Note, for instance, that the highest overall accuracy of M-PSA-HNN-MRF was lower than that of M-PSA and M-PSA-HNN. Thus a multiple classifier system using only a subset of the classification methods can be more accurate than one using the whole set available.

\subsection{AVIRIS hyperspectral image}

\subsubsection{Overview}

A set of analyses based on a real remotely sensed data set were undertaken. This research used an AVIRIS image to map land-cover with the result validated against reference data obtained from visual interpretation of imagery in Google Earth.

\subsubsection{Data}

An AVIRIS image acquired on 11 June 2008 comprising 224 spectral bands with a spatial resolution of $17 \mathrm{~m}$ for a test site centred on the airport located in Moffett Field, San Francisco Bay, USA, was used (figure 4, 37² $24^{\prime} 54^{\prime \prime} \mathrm{N}$ and $122^{\circ} 02^{\prime} 54^{\prime \prime} \mathrm{W}$ ). The focus was on a $180 \times 70$ pixel subset of the imagery for which a reference map was generated using a $900 \times 350$ pixel fine spatial resolution image available in Google Earth acquired on 13 October 2008. The Google Earth image was geo-registered to 
the AVIRIS image (root mean squared error was $4.12 \mathrm{~m}$ ). The scale factor was set $s=5$. The image contained 4 land-cover classes, namely, water, grass, dark surface, and white surface. The endmember signatures in the AVIRIS image were selected using $\mathrm{N}$-finder algorithm (Winter 1999). According to the geometry of convex sets, the $\mathrm{N}$-finder is based on the fact that in $p$ spectral dimensions, the $p$-volume contained by a simplex formed of the purest pixels is larger than any other volume formed from any other combination of pixels. The multiple endmember spectral mixture analysis was applied to generate land-cover class areal proportion images.

\#Insert Figure 4 here\#

\subsubsection{Results and discussion}

The SRMs obtained from the analyses of the AVIRIS image are shown in figure 4. In the maps obtained from the PSA, HNN and MRF, many speckle-like artefacts (examples highlighted in the red circles in figure 4) were observed. This is because the fractional covers, which were absent to a pixel but allocated by soft classification, were maintained in the PSA and were partly smoothed in the HNN and MRF. Some speckle-like artefacts were still found in the HNN and MRF. This because the class proportion RMSE value was 0.2302 for the spectral unmixing output which was very large. Scatter plots of the reference and unmixed class areal proportions are shown in figure 5. The scatter plots indicate that there was obvious overestimation and underestimation in the grass area, and obvious underestimation in the light surface area. In the PSA in figure 4, the speckle-like artefacts in the region A were due to the underestimation of the grass fraction, and the speckle-like artefacts in the regions $\mathrm{B}$ and $\mathrm{C}$ were due to the underestimation of the light surface fraction. There are more fractional covers represented as large speckle-like artefacts in the coarse pixels in the AVIRIS image than in the simulated image; the spatial smoothing effect in the HNN and MRF, which could eliminate isolated pixels, could not eliminate all the large speckle-like artefacts (examples are highlighted in the red circles in figure 4). The speckle-like artefacts in the red circles were not reduced obviously in the maps obtained from the pixel-based M-PSA, M-HNN and M-MRF, because the pixels were 
frequently labelled as speckle-like artefacts in different maps available to the M-SRM. By contrast, the map generated from the context-based M-PSA, M-HNN and M-MRF eliminated most of the speckle-like artefacts due to the spatial smoothing effect from the neighbourhood sub-pixels. Similarly, the maps obtained from the pixel-based M-PSA-HNN, M-PSA-MRF, M-HNN-MRF and M-PSA-HNN-MRF contained many speckle-like artefacts, whereas the maps obtained from the context-based M-PSA-HNN, M-PSA-MRF, M-HNN-MRF and M-PSA-HNN-MRF eliminated the speckle-like artefacts and contained smoothed class boundaries.

More accurate sub-pixel maps were obtained from the M-SRM relative to the single algorithm SRM analyses. The highest overall accuracies of PSA, HNN and MRF were $88.89 \%, 93.81 \%$ and $82.70 \%$, respectively and increased to $89.99 \%$, $94.05 \%$ and $82.92 \%$ respectively for the pixel-based M-PSA, M-HNN and M-MRF, and increased to $95.06 \%, 95.37 \%$ and $85.56 \%$ respectively for the context-based M-PSA, M-HNN and M-MRF. The highest overall accuracies of the context-based M-SRM were much higher than that of the pixel-based M-SRM for each M-SRM. Higher overall accuracies were found for the context-based M-SRM with $W=9$ and $r \geq 3$, which is different from the results obtained with the simulated data. This is because the spectral unmixing error was larger in the AVIRIS image experiment than the simulated image experiment, and the maps obtained from the PSA, HNN and MRF for the AVIRIS image contained more large speckle-like artefacts. The context-based M-SRM with larger $W$ and $r$, which indicates a larger neighbourhood window size that explores more sub-pixels with local spatial autocorrelation and a higher spatial autocorrelation between distant sub-pixels in the $W \times W$ neighbourhood window, eliminated most of the artefacts more efficiently.

In terms of the overall classification accuracy, the map obtained from the MRF was less accurate than that from the PSA and HNN. The highest overall accuracy of M-MRF was lower than that of M-PSA and M-HNN, and the highest overall accuracies of M-PSA-MRF, M-HNN-MRF and M-PSA-HNN-MRF which combined the MRF were lower than that of M-PSA-HNN which excluded the MRF. As with the simulated data, the results showed that M-SRM which combined the whole set of different maps did not always performed better than M-SRM which combined only subset of maps with high accuracies, highlighting the need for care in the selection of algorithms to use within a multi-classifier system. 
\#Insert Tables 5-7 here\#

\section{Conclusion}

The potential to enhance land-cover mapping from remotely sensed data through the combination of multiple sub-pixel maps obtained from a set of SRM analyses was explored. In the multiple SRM approach, M-SRM, each sub-pixel is allocated the class label which is most frequently predicted for it in the SRM outputs that are available. Critically the results of two studies using PSA, HNN and MRF show that the M-SRM approach can increase the accuracy of land-cover maps over that achieved through the conventional use of a single SRM analysis. The land-cover maps generated from the M-SRM were also visually superior to maps from standard single SRM analyses, with fewer speckle-like artefacts and linear features such as paths more fully connected. Given that researchers often run a SRM algorithm several times in order to determine the optimal parameter settings the results show that using, rather than discarding, the outputs of these trial runs can sometimes enhance the accuracy of SRM. The algorithms selected for use in M-SRM also plays a key role in relation to map accuracy. The accuracy of maps obtained from M-SRM that used different algorithms was not always higher than that based on the use of a single algorithm.

The pixel-based M-SRM which labels a sub-pixel based on the classes depicted in the multiple maps for that sub-pixel and the context-based M-SRM which labels a sub-pixel based on the classes depicted in the multiple maps for that sub-pixel and its neighbouring sub-pixels were assessed. The highest overall accuracies of the context-based M-SRM were higher than that of the pixel-based M-SRM for each M-SRM. In addition, the performance of the context-based M-SRM was found to be dependent on the neighbourhood window size used and the magnitude of the parameter $r$ which controls the magnitude of spatial autocorrelations between sub-pixels in the $W \times W$ neighbourhood window. In the context-based M-SRM, a larger $W$ indicates a larger neighbourhood window size that explores more sub-pixels with local spatial autocorrelations, and the spatial autocorrelations between distant sub-pixels in the $W \times W$ neighbourhood window are higher with a larger $r$ than with a smaller $r$. The context-based M-SRM would be expected to better preserve the spatial 
details of land-cover which were not larger than the coarse resolution pixel size with small values of $W$ and $r$. In the simulated image analysis, most land-covers which are smaller than the coarse resolution pixel size were reconstructed with $W<5$ and $r<3$. In addition, the context-based M-SRM would be expected to better eliminate speckle-like artefacts with large values of $W$ and $r$. In the AVIRIS data set the class areal proportion image error was large, and the PSA, HNN and MRF maps contained many large speckle-like artefacts; most of the speckle-like artefacts were eliminated in the context-based M-SRM with $W=9$ and $r \geq 3$. In addition, the selection of the optimal $W$ and $r$ values in the context-based M-SRM may be affected by the geo-reference error between the reference and input images, and the context-based M-SRM performed better with relative smaller $W$ and $r$ values in the simulated image experiment in which the geo-reference root mean squared error was 0 and with relative larger $W$ and $r$ values in the AVIRIS image experiment in which the geo-reference root mean squared error was $4.12 \mathrm{~m}$. A comprehensive study on impact of geo-reference error on M-SRM should be explored in the future.

\section{Acknowledgement}

This work was supported in part by the Natural Science Foundation of China [41301398], the Wuhan ChenGuang Youth Sci.\&Tech. Project [2014072704011254], and the key project from Institute of Geodesy and Geophysics [Y409123012].

\section{References}

Atkinson, P. M. 2005. "Sub-pixel target mapping from soft-classified, remotely sensed imagery." Photogrammetric Engineering and Remote Sensing 71 (7):839-46. doi: 10.14358/PERS.71.7.839.

Atkinson, P. M. 2009. "Issues of uncertainty in super-resolution mapping and their implications for the design of an inter-comparison study." International Journal of Remote Sensing 30 (20):5293-308. doi: 10.1080/01431160903131034.

Benediktsson, J. A., and J. R. Sveinsson. 2003. "Multisource remote sensing data classification based on consensus and pruning." IEEE Transactions on Geoscience and Remote Sensing 41 (4):932-6. doi: 10.1109/tgrs.2003.812000.

Briem, G. J., J. A. Benediktsson, and J. R. Sveinsson. 2002. "Multiple classifiers applied to multisource remote sensing data." IEEE Transactions on Geoscience and Remote Sensing 40 (10):2291-9. doi: 10.1109/tgrs.2002.802476.

Bruzzone, L., R. Cossu, and G. Vernazza. 2004. "Detection of land-cover transitions by combining multidate classifiers." Pattern Recognition Letters 25 (13):1491-500. doi: 
10.1016/j.patrec.2004.06.002.

Doan, H. T. X., and G. M. Foody. 2007. "Increasing soft classification accuracy through the use of an ensemble of classifiers." International Journal of Remote Sensing 28 (20):4609-23. doi: 10.1080/01431160701244872.

Foody, G. M., and H. T. X. Doan. 2007. "Variability in soft classification prediction and its implications for sub-pixel scale change detection and super resolution mapping." Photogrammetric Engineering and Remote Sensing 73 (8):923-33. doi: 10.14358/PERS.73.8.923.

Foody, G. M., A. M. Muslim, and P. M. Atkinson. 2005. "Super-resolution mapping of the waterline from remotely sensed data." International Journal of Remote Sensing 26 (24):5381-92. doi: 10.1080/01431160500213292.

Ge, Y. 2013. "Sub-pixel land-cover mapping with improved fraction images upon multiple-point simulation." International Journal of Applied Earth Observation and Geoinformation 22:115-26. doi: 10.1016/j.jag.2012.04.013.

Ge, Y., Y. H. Chen, S. P. Li, and Y. Jiang. 2014. "Vectorial boundary-based sub-pixel mapping method for remote-sensing imagery." International Journal of Remote Sensing 35 (5):1756-68. doi: 10.1080/01431161.2014.882034.

Ho, T. K., J. J. Hull, and S. N. Srihari. 1994. "Decision combination in multiple classifier systems." IEEE Transactions on Pattern Analysis and Machine Intelligence 16 (1):66-75. doi: 10.1109/34.273716.

Hu, J. L., Y. Ge, Y. H. Chen, and D. Y. Li. 2015. "Super-resolution land cover mapping based on multiscale spatial regularization." IEEE Journal of Selected Topics in Applied Earth Observations and Remote Sensing 8 (5):2031-9. doi: 10.1109/jstars.2015.2399509.

Hu, X. F., and Q. H. Weng. 2011. "Estimating impervious surfaces from medium spatial resolution imagery: a comparison between fuzzy classification and LSMA." International Journal of Remote Sensing 32 (20):5645-63. doi: 10.1080/01431161.2010.507258.

Jin, J., B. Wang, and L. M. Zhang. 2010. "A novel approach based on fisher discriminant null space for decomposition of mixed pixels in hyperspectral imagery." IEEE Geoscience and Remote Sensing Letters 7 (4):699-703. doi: 10.1109/lgrs.2010.2046134.

Kasetkasem, T., M. K. Arora, and P. K. Varshney. 2005. "Super-resolution land cover mapping using a Markov random field based approach." Remote Sensing of Environment 96 (3-4):302-14. doi: 10.1016/j.rse.2005.02.006.

Kavzoglu, T., and I. Colkesen. 2013. "An assessment of the effectiveness of a rotation forest ensemble for land-use and land-cover mapping." International Journal of Remote Sensing 34 (12):4224-41. doi: 10.1080/01431161.2013.774099.

Latif-Shabgahi, G., J. M. Bass, and S. Bennett. 2004. "Taxonomy for software voting algorithms used in safety-critical systems." IEEE Transactions on Reliability 53 (3):319-28. doi: 10.1109/tr.2004.832819.

Li, X. D., Y. Du, and F. Ling. 2012. "Spatially adaptive smoothing parameter selection for Markov random field based sub-pixel mapping of remotely sensed images." International Journal of Remote Sensing 33 (24):7886-901. doi: 10.1080/01431161.2012.703347.

Li, X. D., F. Ling, and Y. Du. 2012. "Super-resolution mapping based on the supervised fuzzy c-means approach." Remote Sensing Letters 3 (6):501-10. doi: 10.1080/01431161.2011.631607.

Lin, X. F., S. Yacoub, J. Burns, and S. Simske. 2003. "Performance analysis of pattern classifier combination by plurality voting." Pattern Recognition Letters 24 (12):1959-69. doi: 
10.1016/s0167-8655(03)00035-7.

Ling, F., Y. Du, X. D. Li, W. B. Li, F. Xiao, and Y. H. Zhang. 2013. "Interpolation-based super-resolution land cover mapping." Remote Sensing Letters 4 (7):629-38. doi: 10.1080/2150704x.2013.781284.

Ling, F., Y. Du, F. Xiao, H. P. Xue, and S. J. Wu. 2010. "Super-resolution land-cover mapping using multiple sub-pixel shifted remotely sensed images." International Journal of Remote Sensing 31 (19):5023-40. doi: 10.1080/01431160903252350.

Ling, F., X. D. Li, F. Xiao, and Y. Du. 2014. "Superresolution land cover mapping using spatial regularization." IEEE Transactions on Geoscience and Remote Sensing 52 (7):4424-39. doi: 10.1109/Tgrs.2013.2281992.

Makido, Yasuyo K., Joseph P. Messina, and Ashton M. Shortridge. 2008. "Exploring the impacts of pseudo-random number generators on sub-pixel classification." Giscience \& Remote Sensing 45 (4):471-89. doi: 10.2747/1548-1603.45.4.471.

Parhami, B. 1994. "Voting algorithms." IEEE Transactions on Reliability 43 (4):617-29. doi: 10.1109/24.370218.

Settle, J. J., and N. A. Drake. 1993. "Linear mixing and the estimation of ground cover proportions." International Journal of Remote Sensing 14 (6):1159-77. doi: 10.1080/01431169308904402.

Su, Y. F., G. M. Foody, A. M. Muad, and K. S. Cheng. 2012a. "Combining Hopfield Neural Network and Contouring Methods to Enhance Super-Resolution Mapping." IEEE Journal of Selected Topics in Applied Earth Observations and Remote Sensing 5 (5):1403-17. doi: Doi 10.1109/Jstars.2012.2191537

Su, Y. F., G. M. Foody, A. M. Muad, and K. S. Cheng. 2012b. "Combining pixel swapping and contouring methods to enhance super-resolution mapping." IEEE Journal of Selected Topics in Applied Earth Observations and Remote Sensing 5 (5):1428-37. doi: Doi 10.1109/Jstars.2012.2216514.

Tarabalka, Yuliya, Mathieu Fauvel, Jocelyn Chanussot, and Jon Atli Benediktsson. 2010. "SVM- and MRF-based method for accurate classification of hyperspectral images." IEEE Geoscience and Remote Sensing Letters 7 (4):736-40. doi: 10.1109/lgrs.2010.2047711.

Tatem, A. J., H. G. Lewis, P. M. Atkinson, and M. S. Nixon. 2001. "Super-resolution target identification from remotely sensed images using a Hopfield neural network." IEEE Transactions on Geoscience and Remote Sensing 39 (4):781-96. doi: 10.1109/36.917895.

Tolpekin, V. A., and A. Stein. 2009. "Quantification of the effects of land-cover-class spectral separability on the accuracy of Markov-random-field-based superresolution mapping." IEEE Transactions on Geoscience and Remote Sensing 47 (9):3283-97. doi: 10.1109/Tgrs.2009.2019126.

Wang, Q. M., L. G. Wang, and D. F. Liu. 2012. "Particle swarm optimization-based sub-pixel mapping for remote-sensing imagery." International Journal of Remote Sensing 33 (20):6480-96. doi: 10.1080/01431161.2012.690541.

Winter, M. E. 1999. "N-FINDR: an algorithm for fast autonomous spectral end-member determination in hyperspectral data." In Imaging Spectrometry V, edited by M. R. Descour and S. S. Shen, 266-75. 
Table 1. M-SRM names and definitions.

\begin{tabular}{|c|c|}
\hline M-SRM analysis & Definition \\
\hline M-SRM & The multiple SRM algorithm \\
\hline $\begin{array}{l}\text { Pixel-based } \\
\text { M-SRM }\end{array}$ & $\begin{array}{l}\text { M-SRM that determines a sub-pixel label based on the classes } \\
\text { depicted in the multiple maps for that sub-pixel }\end{array}$ \\
\hline $\begin{array}{l}\text { Context-based } \\
\text { M-SRM }\end{array}$ & $\begin{array}{l}\text { M-SRM that determines a sub-pixel label based on the classes } \\
\text { depicted in the multiple maps for that sub-pixel and its } \\
\text { neighbouring sub-pixels }\end{array}$ \\
\hline M-PSA & M-SRM that combines multiple PSA outputs \\
\hline M-HNN & M-SRM that combines multiple HNN outputs \\
\hline M-MRF & M-SRM that combines multiple MRF outputs \\
\hline M-PSA-HNN & M-SRM that combines multiple PSA and HNN outputs \\
\hline M-PSA-MRF & M-SRM that combines multiple PSA and MRF outputs \\
\hline M-HNN-MRF & M-SRM that combines multiple HNN and MRF outputs \\
\hline M-PSA-HNN-MRF & M-SRM that combines multiple PSA, HNN, and MRF outputs \\
\hline
\end{tabular}


Table 2. The accuracies of the simulated image experiment, including the mean values, highest value and standard deviations of overall accuracies (\%) of PSA, HNN and MRF.

\begin{tabular}{cccc}
\hline \multirow{2}{*}{ Method } & \multicolumn{3}{c}{ Overall accuracy $(\%)$} \\
\cline { 2 - 4 } & Mean value & Highest value & Standard deviation \\
\hline PSA & 88.73 & 90.14 & 1.08 \\
HNN & 88.11 & 89.15 & 0.78 \\
MRF & 88.31 & 89.76 & 0.94 \\
\hline
\end{tabular}


Table 3. The accuracies of the simulated image experiment, including the overall accuracies (\%) of M-PSA (M-SRM that combines multiple PSA outputs), M-HNN (M-SRM that combines multiple HNN outputs), and M-MRF (M-SRM that combines multiple MRF outputs). The highest M-SRM overall accuracies were highlighted in bold.

\begin{tabular}{|c|c|c|c|c|c|c|c|c|c|c|c|c|c|}
\hline \multirow{2}{*}{\multicolumn{2}{|c|}{ Experimental set up }} & \multicolumn{12}{|c|}{ Overall accuracy (\%) } \\
\hline & & \multicolumn{4}{|c|}{ M-PSA } & \multicolumn{4}{|c|}{ M-HNN } & \multicolumn{4}{|c|}{ M-MRF } \\
\hline $\begin{array}{c}\text { Pixel-based } \\
\text { M-SRM }\end{array}$ & $W=1$ & \multicolumn{4}{|c|}{91.52} & \multicolumn{4}{|c|}{91.38} & \multicolumn{4}{|c|}{91.04} \\
\hline \multirow{5}{*}{$\begin{array}{l}\text { Context } \\
\text {-based } \\
\text { M-SRM }\end{array}$} & & $r=1$ & $r=2$ & $r=3$ & $r=10$ & $r=1$ & $r=2$ & $r=3$ & $r=10$ & $r=1$ & $r=2$ & $r=3$ & $r=10$ \\
\hline & $W=3$ & 92.20 & 92.27 & 92.26 & 92.25 & 91.58 & 91.56 & 91.55 & 91.55 & 91.27 & 91.22 & 91.24 & 91.25 \\
\hline & $W=5$ & 92.19 & 92.11 & 91.91 & 91.69 & 91.59 & 91.31 & 91.05 & 90.71 & 91.23 & 90.95 & 90.67 & 90.36 \\
\hline & $W=7$ & 92.20 & 91.99 & 91.29 & 89.77 & 91.60 & 91.06 & 90.07 & 88.26 & 91.22 & 90.72 & 89.59 & 87.54 \\
\hline & $W=9$ & 92.20 & 91.98 & 90.58 & 83.56 & 91.60 & 91.03 & 89.36 & 82.88 & 91.22 & 90.64 & 88.59 & 82.42 \\
\hline
\end{tabular}


Table 4.The overall accuracies (\%) of M-PSA-HNN, M-PSA-MRF, M-HNN-MRF, M-PSA-HNN-MRF for simulated image experiment. The highest M-SRM overall accuracies for each multiple classification analysis were highlighted in bold.

\begin{tabular}{|c|c|c|c|c|c|c|c|c|c|}
\hline \multirow{2}{*}{\multicolumn{2}{|c|}{ Experimental set up }} & \multicolumn{8}{|c|}{ Overall accuracy $(\%)$} \\
\hline & & \multicolumn{4}{|c|}{ M-PSA-HNN } & \multicolumn{4}{|c|}{ M-PSA-MRF } \\
\hline $\begin{array}{c}\text { Pixel-based } \\
\text { M-SRM }\end{array}$ & $W=1$ & \multicolumn{4}{|c|}{92.15} & \multicolumn{4}{|c|}{92.03} \\
\hline \multirow{5}{*}{$\begin{array}{l}\text { Context } \\
\text {-based } \\
\text { M-SRM }\end{array}$} & & $r=1$ & $r=2$ & $r=3$ & $r=10$ & $r=1$ & $r=2$ & $r=3$ & $r=10$ \\
\hline & $W=3$ & 92.34 & 92.28 & 92.25 & 92.20 & 92.02 & 92.11 & 92.06 & 92.06 \\
\hline & $W=5$ & 92.32 & 92.04 & 91.65 & 91.49 & 92.03 & 91.76 & 91.54 & 91.32 \\
\hline & $W=7$ & 92.33 & 91.80 & 90.87 & 89.06 & 92.03 & 91.61 & 90.63 & 88.83 \\
\hline & $W=9$ & 92.33 & 91.77 & 90.07 & 83.25 & 92.03 & 91.60 & 89.84 & 82.87 \\
\hline & & \multicolumn{4}{|c|}{ M-HNN-MRF } & \multicolumn{4}{|c|}{ M-PSA-HNN-MRF } \\
\hline $\begin{array}{c}\text { Pixel-based } \\
\text { M-SRM }\end{array}$ & $W=1$ & \multicolumn{4}{|c|}{91.59} & \multicolumn{4}{|c|}{92.11} \\
\hline \multirow{5}{*}{$\begin{array}{l}\text { Context } \\
\text {-based } \\
\text { M-SRM }\end{array}$} & & $r=1$ & $r=2$ & $r=3$ & $r=10$ & $r=1$ & $r=2$ & $r=3$ & $r=10$ \\
\hline & $W=3$ & 91.68 & 91.60 & 91.57 & 91.56 & 92.12 & 92.06 & 92.04 & 92.06 \\
\hline & $W=5$ & 91.67 & 91.29 & 90.93 & 90.63 & 92.11 & 91.68 & 91.48 & 91.27 \\
\hline & $W=7$ & 91.67 & 91.03 & 89.97 & 89.93 & 92.11 & 91.56 & 90.55 & 88.61 \\
\hline & $W=9$ & 91.67 & 90.95 & 89.00 & 82.65 & 92.11 & 91.51 & 89.66 & 82.92 \\
\hline
\end{tabular}


Table 5. The accuracies of the AVIRIS image experiment, including the mean values, highest value and standard deviations of overall accuracies (\%) of PSA, HNN and MRF.

\begin{tabular}{cccc}
\hline \multirow{2}{*}{ Method } & \multicolumn{3}{c}{ Overall accuracy $(\%)$} \\
\cline { 2 - 4 } & Mean value & Highest value & Standard deviation \\
\hline PSA & 87.63 & 88.89 & 0.65 \\
HNN & 93.19 & 93.81 & 0.43 \\
MRF & 82.21 & 82.70 & 0.46 \\
\hline
\end{tabular}


Table 6. The accuracies of the AVIRIS image experiment, including the overall accuracies (\%) of M-PSA, M-HNN and M-MRF. The highest M-SRM overall accuracies were highlighted in bold.

\begin{tabular}{|c|c|c|c|c|c|c|c|c|c|c|c|c|c|}
\hline \multirow{2}{*}{\multicolumn{2}{|c|}{ Experimental set up }} & \multicolumn{12}{|c|}{ Overall accuracy $(\%)$} \\
\hline & & \multicolumn{4}{|c|}{ M-PSA } & \multicolumn{4}{|c|}{ M-HNN } & \multicolumn{4}{|c|}{ M-MRF } \\
\hline $\begin{array}{c}\text { Pixel-based } \\
\text { M-SRM }\end{array}$ & $W=1$ & \multicolumn{4}{|c|}{89.99} & \multicolumn{4}{|c|}{94.05} & \multicolumn{4}{|c|}{82.92} \\
\hline \multirow{5}{*}{$\begin{array}{l}\text { Context } \\
\text {-based } \\
\text { M-SRM }\end{array}$} & & $r=1$ & $r=2$ & $r=3$ & $r=10$ & $r=1$ & $r=2$ & $r=3$ & $r=10$ & $r=1$ & $r=2$ & $r=3$ & $r=10$ \\
\hline & $W=3$ & 91.66 & 92.12 & 92.20 & 92.21 & 94.27 & 94.34 & 94.35 & 94.36 & 83.66 & 83.82 & 83.85 & 83.86 \\
\hline & $W=5$ & 91.75 & 92.95 & 93.28 & 93.53 & 94.29 & 94.55 & 94.65 & 94.74 & 83.70 & 84.17 & 84.35 & 84.49 \\
\hline & $W=7$ & 91.75 & 93.25 & 93.92 & 94.44 & 94.29 & 94.65 & 94.92 & 95.37 & 83.70 & 84.31 & 84.68 & 85.03 \\
\hline & $W=9$ & 91.75 & 93.31 & 94.26 & 95.06 & 94.29 & 94.67 & 95.04 & 95.31 & 83.70 & 84.34 & 85.56 & 85.51 \\
\hline
\end{tabular}


Table 7.The overall accuracies (\%) of M-PSA-HNN, M-PSA-MRF, M-HNN-MRF, M-PSA-HNN-MRF for AVIRIS image experiment. The highest M-SRM overall accuracies for each multiple classification analysis were highlighted in bold.

\begin{tabular}{|c|c|c|c|c|c|c|c|c|c|}
\hline \multirow{2}{*}{\multicolumn{2}{|c|}{ Experimental set up }} & \multicolumn{8}{|c|}{ Overall accuracy (\%) } \\
\hline & & \multicolumn{4}{|c|}{ M-PSA-HNN } & \multicolumn{4}{|c|}{ M-PSA-MRF } \\
\hline Pixel-based & $W=1$ & \multicolumn{4}{|c|}{93.57} & \multicolumn{4}{|c|}{87.23} \\
\hline \multirow{5}{*}{$\begin{array}{l}\text { Context } \\
\text {-based } \\
\text { M-SRM }\end{array}$} & & $r=1$ & $r=2$ & $r=3$ & $r=10$ & $r=1$ & $r=2$ & $r=3$ & $r=10$ \\
\hline & $W=3$ & 93.91 & 94.00 & 94.02 & 94.03 & 87.75 & 87.94 & 87.98 & 88.00 \\
\hline & $W=5$ & 93.93 & 94.30 & 94.41 & 94.49 & 87.78 & 88.35 & 88.53 & 88.68 \\
\hline & $W=7$ & 93.93 & 94.41 & 94.69 & 94.97 & 87.78 & 88.48 & 88.87 & 89.29 \\
\hline & $W=9$ & 93.93 & 94.44 & 94.87 & 95.30 & 87.78 & 88.51 & 89.07 & 89.80 \\
\hline & & \multicolumn{4}{|c|}{ M-HNN-MRF } & \multicolumn{4}{|c|}{ M-PSA-HNN-MRF } \\
\hline Pixel-based & $W=1$ & \multicolumn{4}{|c|}{89.64} & \multicolumn{4}{|c|}{91.76} \\
\hline \multirow{5}{*}{$\begin{array}{l}\text { Context } \\
\text {-based } \\
\text { M-SRM }\end{array}$} & & $r=1$ & $r=2$ & $r=3$ & $r=10$ & $r=1$ & $r=2$ & $r=3$ & $r=10$ \\
\hline & $W=3$ & 90.14 & 90.18 & 90.18 & 90.18 & 91.91 & 91.88 & 91.87 & 91.87 \\
\hline & $W=5$ & 90.23 & 90.40 & 90.47 & 90.51 & 91.93 & 92.00 & 92.03 & 92.06 \\
\hline & $W=7$ & 90.23 & 90.49 & 90.65 & 90.80 & 91.93 & 92.07 & 92.23 & 92.37 \\
\hline & $W=9$ & 90.23 & 90.52 & 91.11 & 91.01 & 91.93 & 92.10 & 92.61 & 92.36 \\
\hline
\end{tabular}




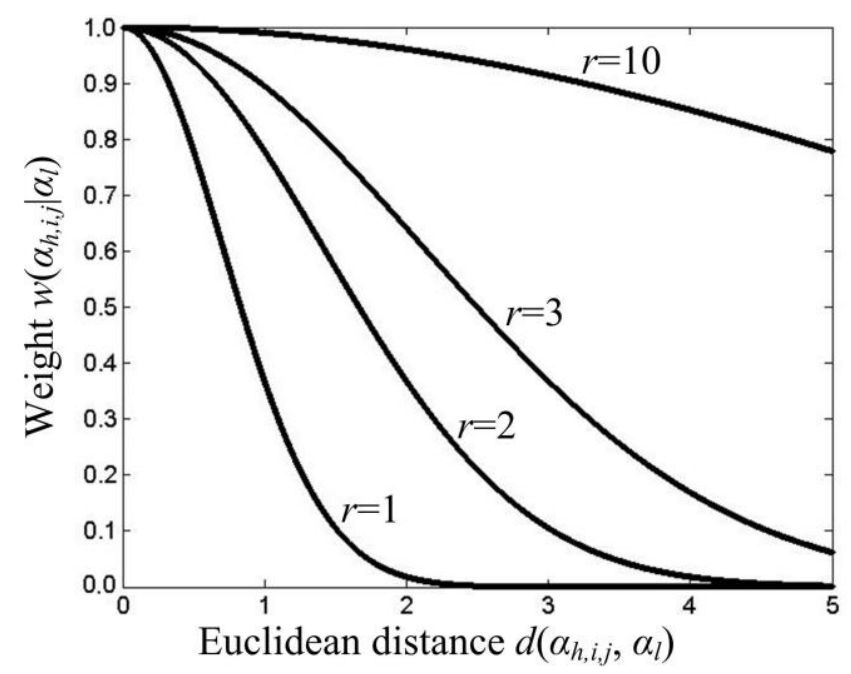

Figure 1 The relative magnitude of the weight $w\left(\alpha_{h, i, j} \mid \alpha_{l}\right)$ with Euclidean distance $d\left(\alpha_{h, i, j}, \alpha_{l}\right)$ according to different range value $r$ in the $W \times W$ neighbourhood window centred on $\alpha_{h, i, j}$ in the context-based M-SRM. The weight $w\left(\alpha_{h, i, j} \mid \alpha_{l}\right)$ decreases most sharply with the increase of $d\left(\alpha_{h, i, j}, \alpha_{l}\right)$ for small values of $r$. 


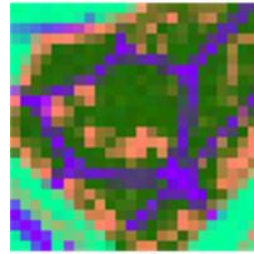

(a)

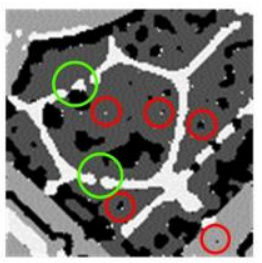

(d)

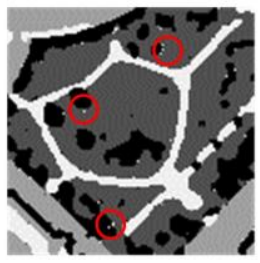

(g)

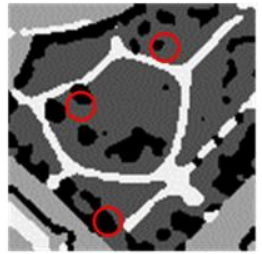

(j)

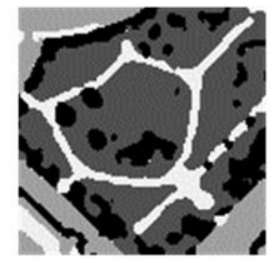

(m)

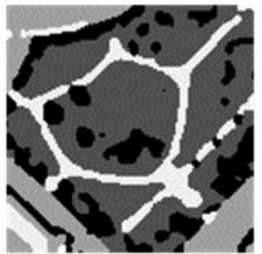

(q)

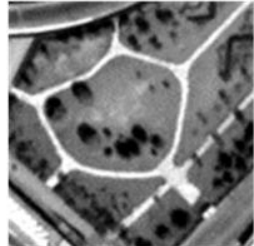

(b)

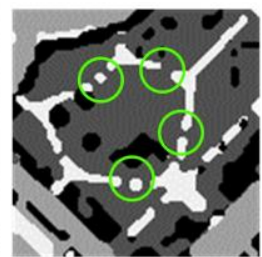

(e)

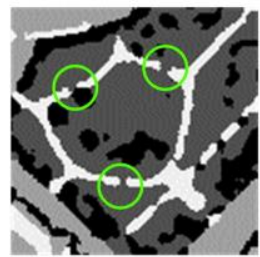

(h)

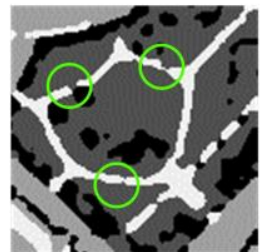

(k)

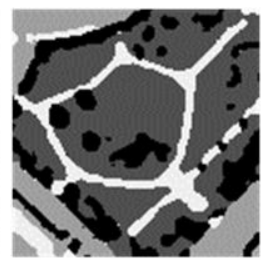

(n)

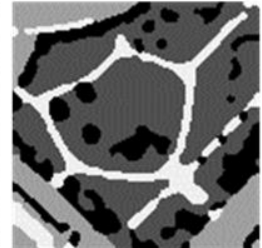

(r)

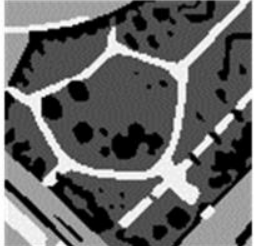

(c)

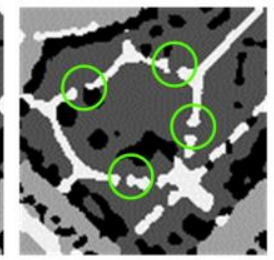

(f)

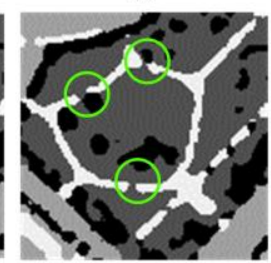

(i)

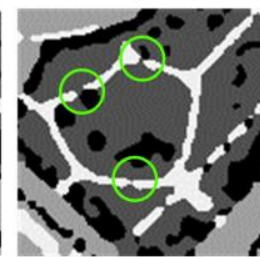

(l)

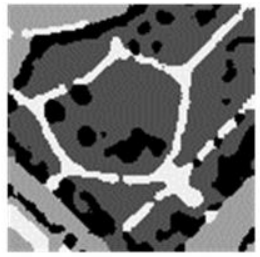

(o)

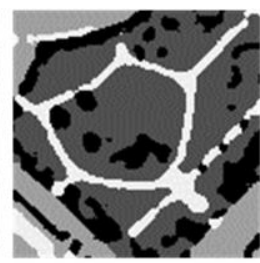

(p)

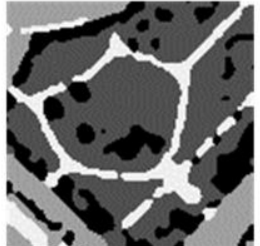

(s)

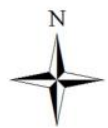

Legend
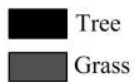

$\square$ Bare earth

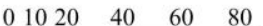

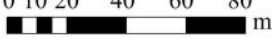

Figure 2. Reference and result maps for the simulated image experiment. (a) Simulated multi-spectral image (band 1-2-3), (b) panchromatic image, $(c)$ reference map, $(d)$ PSA with the highest overall accuracy, $(e) \mathrm{HNN}$ with the highest overall accuracy, $(f)$ MRF with the highest overall accuracy, $(g)$ the pixel-based M-PSA, $(h)$ the pixel-based M-HNN, $(i)$ the pixel-based M-MRF, $(j)$ the context-based M-PSA $(w=3, r=2),(k)$ the context-based M-HNN $(w=7, r=1),(l)$ the context-based M-MRF $(w=3, r=1),(m)$ the pixel-based M-PSA-HNN, $(n)$ the pixel-based M-PSA-MRF, $(o)$ the pixel-based M-HNN-MRF, $(p)$ the pixel-based M-PSA-HNN-MRF, $(q)$ the context-based M-PSA-HNN $(w=3, r=1), \quad(r)$ the context-based M-PSA-MRF $(w=3, r=2),(s)$ the context-based M-HNN-MRF $(w=3, r=1),(t)$ the context-based M-PSA-HNN-MRF $(w=3, r=1)$. 


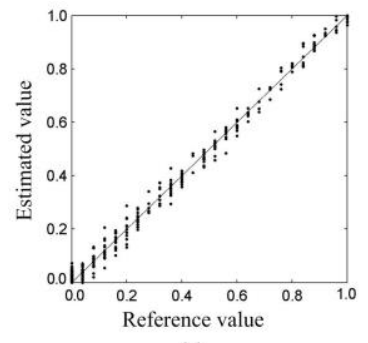

(a)

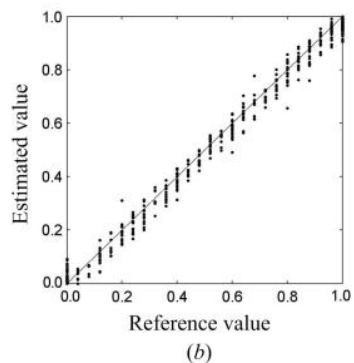

(b)

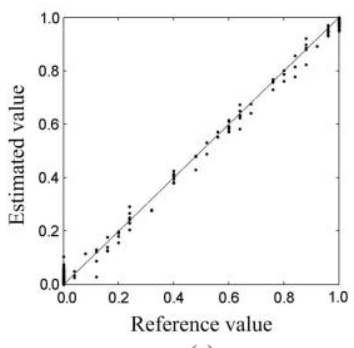

(c)

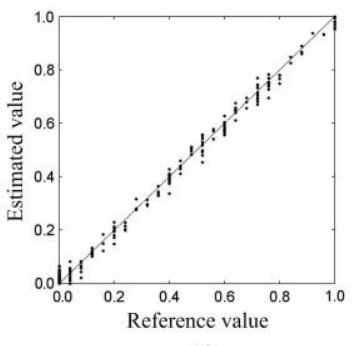

(d)

Figure 3. Scatter plots of linear mixture model accuracy assessment for the simulated image experiment. $(a)$ tree, $(b)$ grass, $(c)$ bare earth, $(d)$ path. 


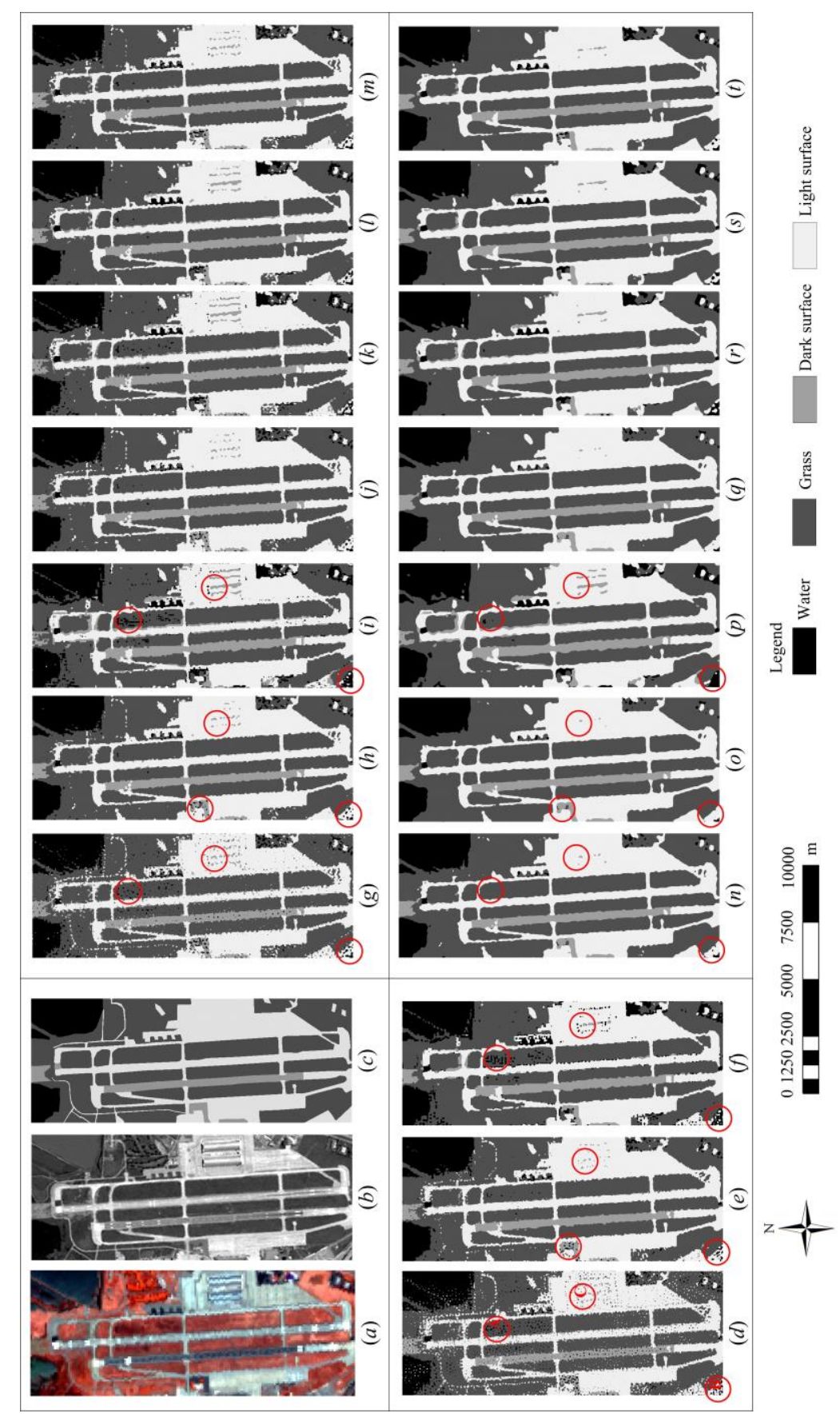

Figure 4. Reference and result maps for the AVIRIS image experiment in Moffett Field site $\left(37^{\circ} 24^{\prime} 54^{\prime \prime} \mathrm{N}\right.$ and $\left.122^{\circ} 02^{\prime} 54^{\prime \prime} \mathrm{W}\right)$. (a) AVIRIS hyperspectral image (band 40-20-15), (b) Google earth image, (c) reference map, (d) PSA with the highest overall accuracy, $(e) \mathrm{HNN}$ with the highest overall accuracy, $(f) \mathrm{MRF}$ with the highest overall accuracy, $(g)$ the pixel-based M-PSA, $(h)$ the pixel-based M-HNN, $(i)$ the pixel-based M-MRF, $(j)$ the pixel-based M-PSA-HNN, $(k)$ the pixel-based M-PSA-MRF, $(l)$ the pixel-based M-HNN-MRF, $(m)$ the pixel-based M-PSA-HNN-MRF, $(n)$ the context-based M-PSA $(w=9, r=10),(o)$ the context-based M-HNN $(w=7, r=10),(p)$ the context-based M-MRF $(w=9, r=3),(q)$ the context-based M-PSA-HNN $(w=9, r=10),(r)$ the context-based M-PSA-MRF $(w=9, r=10),(s)$ the context-based M-HNN-MRF $(w=9, r=3),(t)$ the context-based M-PSA-HNN-MRF $(w=9, r=3)$. 

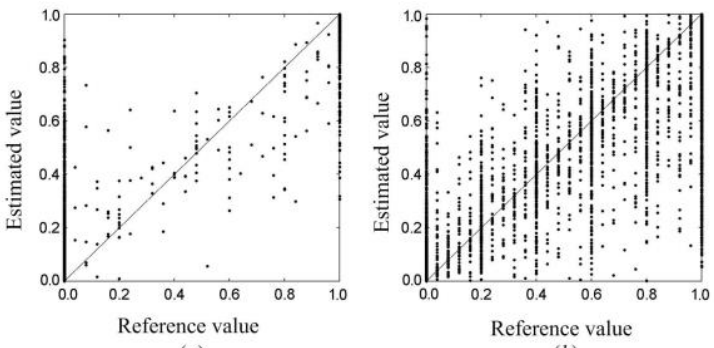

(a)

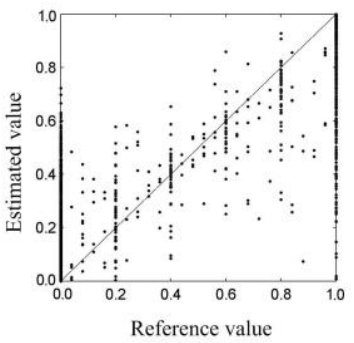

(c)

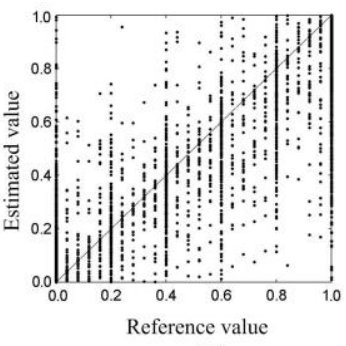

(d)

Figure 5. Scatter plots of multiple endmember spectral mixture analysis accuracy assessment for the AVIRIS image experiment. $(a)$ water, $(b)$ grass, $(c)$ dark surface, $(d)$ light surface. 